\title{
ON A DYNAMIC GAME PROBLEM WITH AN INDECOMPOSABLE SET OF DISTURBANCES ${ }^{1}$
}

\author{
Dmitriy A. Serkov \\ Krasovskii Institute of Mathematics and Mechanics, \\ Ural Branch of the Russian Academy of Sciences, \\ 16 S. Kovalevskaya Str., Ekaterinburg, 620990, Russia \\ serkov@imm.uran.ru
}

\begin{abstract}
For an abstract dynamic system, a game problem of retention of the motions in a given set of the motion histories is considered. The case of an indecomposable set of disturbances is studied. The set of successful solvability and a construction of a resolving quasistrategy based on the method of programmed iterations is proposed.
\end{abstract}

Keywords: Indecomposable disturbances, Quasistrategy, Retention problem.

\section{Introduction}

The theory and methods of solving control problems are widely developed in the case when the instantaneous (geometric) restrictions in couple with measurability are the only claim describing the set of admissible disturbances. For these important problems, such a fundamental results as the theorem on the alternative and the extreme aiming method are established (see [5] and the references therein). This kind of restrictions imply the decomposability property [7] of the set of admissible disturbances. In control problems, this property is known as the possibility to "glue" any admissible disturbances at any time for composing a new admissible disturbance. On the other hand, a notable family of control problems is characterized by the absence of this property: typical cases are given by the systems with continuous or constant disturbances.

In the present paper we consider a dynamic control problem with an indecomposable set of disturbances. The consideration is carried out on the example of a retention problem - a simple case of the positional differential game. We search a solution of the problem in a set of quasistrategies. The description of controlled system is given in an abstract form and, in general, follows the scheme [9]. The proposed solution is based on the method of programmed iterations (see [1] and the references therein; see also [9]). The formalization studied lies in direction of the problems that use an additional information on the functional properties of the set of disturbances (see, e.g., [4, 6]). The absence of topological requirements on the elements of the retention problem is compensated by an increasing of the iterations "number" [8]. As usual in the abstract setting, the control time "interval" is not assumed to be bounded or connected.

\footnotetext{
${ }^{1}$ The reported study was funded by RFBR, project number 19-01-00573.
} 


\section{Problem statement}

\subsection{Dynamic system}

Denote by $\mathcal{P}(T)$ (by $\mathcal{P}^{\prime}(T)$ ) all (all non-empty) subsets of the set $T$. For non-empty sets $A$ and $B$, let $B^{A}$ be the set of all mappings defined on the set $A$ with values in the set $B$. If, in addition, $f \in B^{A}$ and $C \in \mathcal{P}^{\prime}(A)$, then $(f \mid C) \in B^{C}$ denotes the restriction of the mapping $f$ to the set $C$ : $(f \mid C)(x) \triangleq f(x) \forall x \in C$. In case when $F \in \mathcal{P}^{\prime}\left(B^{A}\right)$, we denote $(F \mid C) \triangleq\{(f \mid C): f \in F\}$.

Choose and fix a non-empty subset $\mathbf{I}$ of real numbers $\mathbb{R}$ as an analogue of a time interval. Non-empty sets $\mathbf{X}$ and $\mathbf{Y}$ specify the ranges of the spatial variables and the disturbance values respectively. If $t \in \mathbf{I}$, then we denote $\mathbf{I}^{t} \triangleq\{\xi \in \mathbf{I} \mid \xi \leq t\}$ and $\mathbf{I}_{t} \triangleq\{\xi \in \mathbf{I} \mid \xi \geq t\}$. Let $\mathbf{C} \in \mathcal{P}^{\prime}\left(\mathbf{X}^{\mathbf{I}}\right)$ and $\Omega \in \mathcal{P}^{\prime}\left(\mathbf{Y}^{\mathbf{I}}\right)$ be the sets of admissible trajectories and disturbances respectively. Denote by $\mathcal{D} \triangleq \mathbf{I} \times \mathbf{C} \times \Omega$ the state space of the controlled process. For any $t \in \mathbf{I}, x \in \mathbf{C}$, we denote $\mathrm{Z}_{0}\left(x \mid \mathbf{I}^{t}\right) \triangleq\left\{x^{\prime} \in \mathbf{C} \mid\left(x^{\prime} \mid \mathbf{I}^{t}\right)=\left(x \mid \mathbf{I}^{t}\right)\right\}$.

As an analogue of the dynamic system, we fix a mapping $\mathcal{S}: \mathcal{D} \mapsto \mathcal{P}^{\prime}(\mathbf{C})$ such that $\forall t \in \mathbf{I}$, $\forall \tau \in \mathbf{I}_{t} \forall x, x^{\prime} \in \mathbf{C}$ and $\forall \omega, \omega^{\prime} \in \Omega$

$$
\begin{gathered}
\mathcal{S}(t, x, \omega) \in \mathcal{P}^{\prime}\left(\mathrm{Z}_{0}\left(x \mid \mathbf{I}^{t}\right)\right) \\
\left(\left(x \mid \mathbf{I}^{t}\right)=\left(x^{\prime} \mid \mathbf{I}^{t}\right)\right) \Rightarrow\left(\mathcal{S}(t, x, \omega)=\mathcal{S}\left(t, x^{\prime}, \omega\right)\right) \\
(h \in \mathcal{S}(t, x, \omega)) \Rightarrow(h \in \mathcal{S}(\tau, h, \omega)) \\
\left(\left(\mathcal{S}(t, x, \omega) \mid \mathbf{I}^{\tau}\right)=\left(\mathcal{S}\left(t, x, \omega^{\prime}\right) \mid \mathbf{I}^{\tau}\right) \&(h \in \mathcal{S}(t, x, \omega)) \&\left(h^{\prime} \in \mathcal{S}\left(\tau, h, \omega^{\prime}\right)\right)\right) \Rightarrow\left(h^{\prime} \in \mathcal{S}\left(t, x, \omega^{\prime}\right)\right) .
\end{gathered}
$$

Thus, for every $(t, x, \omega) \in \mathcal{D}, \mathcal{S}(t, x, \omega)$ denotes the set of all trajectories of the system (1.1)-(1.4) corresponding to the history $x$ up to the "moment" $t$ and to the disturbance realization $\omega$ after $t$.

Choose and fix some initial history $\left(t_{0}, x_{0}\right) \in \mathbf{I} \times \mathbf{C}$. All further constructions are carried out in order to formulate and solve the retention problem for this initial history. Let us define the set $\mathbf{S P}_{\left(t_{0}, x_{0}\right)} \in \mathcal{P}^{\prime}(\mathcal{D})$ of all the states of the controlled process arising in the system from the initial history $\left(t_{0}, x_{0}\right)$ when all admissible disturbances are implemented:

$$
\mathbf{S P}_{\left(t_{0}, x_{0}\right)} \triangleq\left\{(t, x, \omega) \in \mathcal{D} \mid t \in \mathbf{I}_{t_{0}}\left(x \mid \mathbf{I}^{t}\right) \in\left(\mathcal{S}\left(t_{0}, x_{0}, \omega\right) \mid \mathbf{I}^{t}\right)\right\}
$$

For a state $(t, x, \omega) \in \mathbf{S P}_{\left(t_{0}, x_{0}\right)}$, we determine the set $\Omega(t, x, \omega)$ of all disturbances that are compatible with this state:

$$
\Omega(t, x, \omega) \triangleq\left\{\omega^{\prime} \in \Omega \mid\left(\mathcal{S}\left(t_{0}, x_{0}, \omega\right) \mid \mathbf{I}^{t}\right)=\left(\mathcal{S}\left(t_{0}, x_{0}, \omega^{\prime}\right) \mid \mathbf{I}^{t}\right)\right\} .
$$

So (see (1.2)), we have $\Omega\left(t_{0}, x_{0}, \omega\right)=\Omega$ for all $\omega \in \Omega$.

\subsection{Control procedures and the retention problem}

We assume that for the formation of the trajectories the controlling side uses non-empty-valued and non-anticipatory multifunctions from $\mathcal{P}(\mathbf{C})^{\Omega}$. So, for a state $(t, x, \omega) \in \mathbf{S P}_{\left(t_{0}, x_{0}\right)}$, we determine the set $\mathbb{M}_{(t, x, \omega)}$ of all admissible control procedures — of quasistrategies - as follows:

$$
\begin{gathered}
\mathbb{M}_{(t, x, \omega)} \triangleq\left\{\alpha \in \prod_{\omega^{\prime} \in \Omega(t, x, \omega)} \mathcal{P}^{\prime}\left(\mathcal{S}\left(t, x, \omega^{\prime}\right)\right) \mid \forall \omega_{1}, \omega_{2} \in \Omega(t, x, \omega) \forall \tau \in \mathbf{I}\right. \\
\left.\left(\left(\mathcal{S}\left(t_{0}, x_{0}, \omega_{1}\right) \mid \mathbf{I}^{\tau}\right)=\left(\mathcal{S}\left(t_{0}, x_{0}, \omega_{2}\right) \mid \mathbf{I}^{\tau}\right)\right) \Rightarrow\left(\left(\alpha\left(\omega_{1}\right) \mid \mathbf{I}^{\tau}\right)=\left(\alpha\left(\omega_{2}\right) \mid \mathbf{I}^{\tau}\right)\right)\right\} .
\end{gathered}
$$


Let the set $\mathbf{D} \in \mathcal{P}^{\prime}(\mathbf{I} \times \mathbf{C})$, which describes the phase constraints, satisfies the conditions

$$
\left(t_{0}, x_{0}\right) \in \mathbf{D}, \quad((t, x) \in \mathbf{D}) \Rightarrow\left(\{t\} \times \mathrm{Z}_{0}\left(x \mid \mathbf{I}^{t}\right) \subset \mathbf{D}\right) .
$$

On the basis of $\mathbf{D}$, we define the set $\mathcal{N}$ in the following way:

$$
\mathcal{N} \triangleq(\mathbf{D} \times \Omega) \cap \mathbf{S P}_{\left(t_{0}, x_{0}\right)}
$$

and consider the retention of states of the control process in the set $\mathcal{N}$ as the aim of control. Namely, we say that the aim is attainable for the initial state $(t, x, \omega)$ if the inclusions

$$
(\tau, h, \nu) \in \mathcal{N}, \quad \forall \tau \in \mathbf{I}_{t}, \forall h \in \alpha(\nu), \forall \nu \in \Omega(t, x, \omega)
$$

hold for some quasistrategy $\alpha \in \mathbb{M}_{(t, x, \omega)}$. For the initial history $\left(t_{0}, x_{0}\right)$, this definition means that projections of current states of the control process on the set $\mathbf{I} \times \mathbf{C}$ remain in $\mathbf{D}$ for any disturbance $\nu \in \Omega$.

\section{The main results}

\subsection{The programmed absorption operator and its iterations}

For $H \in \mathcal{P}\left(\mathbf{S P}_{\left(t_{0}, x_{0}\right)}\right),(t, x, \omega) \in \mathbf{S P}_{\left(t_{0}, x_{0}\right)}$, and $\nu \in \Omega(t, x, \omega)$, define

$$
\Pi(\nu \mid(t, x, \omega), H) \triangleq\left\{h \in \mathcal{S}(t, x, \nu) \mid(\tau, h, \nu) \in H \quad \forall \tau \in \mathbf{I}_{t}\right\} .
$$

In terms of (2.1), we introduce the operator $\mathbf{A}, \mathbf{A} \in \mathcal{P}\left(\mathbf{S P}_{\left(t_{0}, x_{0}\right)}\right)^{\mathcal{P}\left(\mathbf{S P}_{\left(t_{0}, x_{0}\right)}\right)}$, (the programmed absorption operator) by setting

$$
\mathbf{A}(H) \triangleq\{(t, x, \omega) \in H \mid \Pi(\nu \mid(t, x, \omega), H) \neq \varnothing \quad \forall \nu \in \Omega(t, x, \omega)\}
$$

for any $H \in \mathcal{P}\left(\mathbf{S P}_{\left(t_{0}, x_{0}\right)}\right)$. The definition of $\mathbf{A}$ immediately implies that

$$
\mathbf{A}(H) \subset H .
$$

Then, following the transfinite induction method (see, for example, [3, sec. I.3]), let us introduce $\alpha$-iteration $\mathbf{A}^{\alpha}, \mathbf{A}^{\alpha} \in \mathcal{P}\left(\mathbf{S P}_{\left(t_{0}, x_{0}\right)}\right)^{\mathcal{P}\left(\mathbf{S P}_{\left(t_{0}, x_{0}\right)}\right)}$, of the operator $\mathbf{A}$ for every ordinal $\alpha$. For $\alpha=0$, we assume $\mathbf{A}^{0}(H) \triangleq H, \forall H \in \mathcal{P}\left(\mathbf{S P}_{\left(t_{0}, x_{0}\right)}\right)$; if $\alpha$ has a predecessor (let it be an ordinal $\gamma$ ), we write $\mathbf{A}^{\alpha} \triangleq \mathbf{A} \circ \mathbf{A}^{\gamma} ;$ and, if $\alpha$ is a limit ordinal, let $\mathbf{A}^{\alpha}(H) \triangleq \cap_{\beta \prec \alpha} \mathbf{A}^{\beta}(H), \forall H \in \mathcal{P}\left(\mathbf{S P}_{\left(t_{0}, x_{0}\right)}\right)$. Here, by $\prec$, the strict order relation on the class of ordinals is denoted. Then, according to the transfinite induction principle, $\alpha$-iteration $\mathbf{A}^{\alpha}$ of the operator $\mathbf{A}$ is correctly defined for every ordinal $\alpha$. As a consequence of the definitions and (2.3) (see [8, (4.4)]), we get the following embedding for any ordinal $\alpha$ :

$$
\mathbf{A}^{\alpha}(H) \subset H .
$$

\subsection{Quasistrategies solving the retention problem}

Let us study the issue of solvability of the retention problem in the chosen class of quasistrategies. In the following, let the ordinal $\sigma$ be strictly greater than the cardinality of the set $\mathcal{N}:|\mathcal{N}| \prec \sigma$.

Lemma 1. The inclusions below hold:

$$
\Pi\left(\cdot \mid(t, x, \omega), \mathbf{A}^{\sigma}(\mathcal{N})\right) \in \mathbb{M}_{(t, x, \omega)} \quad \forall(t, x, \omega) \in \mathbf{A}^{\sigma}(\mathcal{N}) .
$$


It follows from lemma 1, (2.4), and definition (2.1), that for any $(t, x, \omega) \in \mathbf{A}^{\sigma}(\mathcal{N})$,

$$
(\tau, h, \nu) \in \mathcal{N} \quad \forall \tau \in \mathbf{I}_{t} \forall h \in \Pi\left(\nu \mid(t, x, \omega), \mathbf{A}^{\sigma}(\mathcal{N})\right) \forall \nu \in \Omega(t, x, \omega) .
$$

Thus, for any $(t, x, \omega) \in \mathbf{A}^{\sigma}(\mathcal{N})$, we have obtained an explicit form of a quasistrategy solving the retention problem in $\mathcal{N}$.

Theorem 1. The following equality holds:

$$
\begin{aligned}
& \mathbf{A}^{\sigma}(\mathcal{N})=\left\{(t, x, \omega) \in \mathcal{N} \mid \exists \alpha \in \mathbb{M}_{(t, x, \omega)}:\right. \\
& \left.(\tau, h, \nu) \in \mathcal{N} \forall \tau \in \mathbf{I}_{t} \forall h \in \alpha(\nu) \forall \nu \in \Omega(t, x, \omega)\right\} .
\end{aligned}
$$

Theorem 1 states that the set $\mathbf{A}^{\sigma}(\mathcal{N})$ is the greatest of the subsets of initial positions from $\mathcal{N}$ that admit a solution of the retention problem in $\mathcal{N}$ in the class of quasistrategies. By Theorem 1 , the original retention problem is solvable if and only if $\left(t_{0}, x_{0}, \omega_{0}\right) \in \mathbf{A}^{\sigma}(\mathcal{N})$ for some $\omega_{0} \in \Omega$; as already mentioned, when it is solvable, the quasi-strategy $\Pi\left(\cdot \mid\left(t_{0}, x_{0}, \omega_{0}\right), \mathbf{A}^{\sigma}(\mathcal{N})\right)$ implements this solution (see Lemma 1).

\section{Proofs of the results}

\subsection{Preliminaries}

We begin with some auxiliary results. Lemma 2 is based on the properties (1.2)-(1.4).

Lemma 2. For any $(t, x, \omega) \in \mathcal{D}, \tau \in \mathbf{I}^{t}$, and $h \in \mathcal{S}(t, x, \omega)$, the equality below is true:

$$
\mathcal{S}(\tau, h, \omega)=\mathcal{S}(t, x, \omega) \cap \mathrm{Z}_{0}\left(h \mid \mathbf{I}^{\tau}\right) .
$$

Lemma 3 follows from the definitions (1.5) and (1.6).

Lemma 3. For any $(t, x, \omega) \in \mathbf{S P}_{\left(t_{0}, x_{0}\right)}, \nu \in \Omega(t, x, \omega)$, and $y \in \mathrm{Z}_{0}\left(x \mid \mathbf{I}^{t}\right)$, the relations $(t, x, \nu),(t, y, \nu),(t, y, \omega) \in \mathbf{S P}_{\left(t_{0}, x_{0}\right)}, \Omega(t, x, \nu)=\Omega(t, y, \nu)=\Omega(t, y, \omega)=\Omega(t, x, \omega)$ are fulfilled.

Lemma 4 is some generalization of the results $[1,2,9]$ on the fixed points of the programmed absorption operator. Here, a lack of topological properties of the operator $\mathbf{A}$ and the set $\mathcal{N}$ is compensated by increasing the countable "number" of iterations up to the ordinal $\sigma$. The proof of Lemma 4 is based on property (2.3) and statement [8, Prop. 2].

Lemma 4. For any $H \in \mathcal{P}\left(\mathbf{S P}_{\left(t_{0}, x_{0}\right)}\right)$ and an ordinal $\alpha,|H| \prec \alpha$, the following equality holds:

$$
\mathbf{A}^{\alpha}(H)=\mathbf{A}\left(\mathbf{A}^{\alpha}(H)\right)
$$

Lemma 5. Let $(\tau, h, \nu),\left(\tau, h^{\prime}, \nu^{\prime}\right) \in \mathbf{S P}_{\left(t_{0}, x_{0}\right)}$ and

$$
\begin{gathered}
\left(h \mid \mathbf{I}^{\tau}\right)=\left(h^{\prime} \mid \mathbf{I}^{\tau}\right), \\
\Omega(\tau, h, \nu)=\Omega\left(\tau, h^{\prime}, \nu^{\prime}\right) .
\end{gathered}
$$

Then, $\left((\tau, h, \nu) \in \mathbf{A}^{\eta}(\mathcal{N})\right) \Leftrightarrow\left(\left(\tau, h^{\prime}, \nu^{\prime}\right) \in \mathbf{A}^{\eta}(\mathcal{N})\right)$ holds for any ordinal $\eta$. 
P r o o f. We show the implication

$$
\left((\tau, h, \nu) \in \mathbf{A}^{\eta}(\mathcal{N})\right) \Rightarrow\left(\left(\tau, h^{\prime}, \nu^{\prime}\right) \in \mathbf{A}^{\eta}(\mathcal{N})\right) .
$$

1. Let $(\tau, h, \nu) \in \mathbf{A}^{0}(\mathcal{N})$ satisfy the conditions of Lemma 5. By definition, we have (see (1.9)) $\mathbf{A}^{0}(\mathcal{N})=\mathcal{N}=\mathbf{S P}_{\left(t_{0}, x_{0}\right)} \cap(\mathbf{D} \times \Omega)$. Then, $(\tau, h) \in \mathbf{D}$. Using (3.3) and (1.8), we derive $\left(\tau, h^{\prime}\right) \in \mathbf{D}$. Since $\left(\tau, h^{\prime}, \nu^{\prime}\right) \in \mathbf{S P}_{\left(t_{0}, x_{0}\right)}$, this implies (see $\left.(1.9)\right)\left(\tau, h^{\prime}, \nu^{\prime}\right) \in \mathcal{N}=\mathbf{A}^{0}(\mathcal{N})$. Suppose in general that the ordinal $\alpha$ is such that for all ordinals $\beta, \beta \prec \alpha$, implication (3.5) holds.

2. If $\alpha$ is a limit ordinal, then by definition, $\mathbf{A}^{\alpha}(\mathcal{N})=\bigcap_{\beta \prec \alpha} \mathbf{A}^{\beta}(\mathcal{N})$. If, in addition, $(\tau, h, \nu) \in$ $\mathbf{A}^{\alpha}(\mathcal{N})$, then $(\tau, h, \nu) \in \mathbf{A}^{\beta}(\mathcal{N})$ for all $\beta \prec \alpha$. Hence, by the induction hypothesis from (3.5), we obtain the inclusions $\left(\tau, h^{\prime}, \nu^{\prime}\right) \in \mathbf{A}^{\beta}(\mathcal{N})$ for all $\beta \prec \alpha$. And, therefore, by the definition of $\mathbf{A}^{\alpha}$, we have the inclusion $\left(\tau, h^{\prime}, \nu^{\prime}\right) \in \mathbf{A}^{\alpha}(\mathcal{N})$.

3. If $\alpha$ has a predecessor (let it be an ordinal $\gamma$ ), then, by definition, $\mathbf{A}^{\alpha}(\mathcal{N})=\mathbf{A}\left(\mathbf{A}^{\gamma}(\mathcal{N})\right.$ ). It follows from the definition of $\mathbf{A}$ (see (2.2)), the inclusion $(\tau, h, \nu) \in \mathbf{A}^{\alpha}(\mathcal{N})$, and induction hypothesis (3.5) that

$$
\begin{gathered}
\left(\tau, h^{\prime}, \nu^{\prime}\right) \in \mathbf{A}^{\gamma}(\mathcal{N}), \\
\forall \eta \in \Omega(\tau, h, \nu) \exists g \in \mathcal{S}(\tau, h, \eta):(\xi, g, \eta) \in \mathbf{A}^{\gamma}(\mathcal{N}) \forall \xi \in \mathbf{I}_{\tau} .
\end{gathered}
$$

From (3.3) (see (1.2)), we obtain $\mathcal{S}(\tau, h, \eta)=\mathcal{S}\left(\tau, h^{\prime}, \eta\right)$ for all $\eta \in \Omega$. Then, in view of (3.4), we can rewrite (3.7) in the following form:

$$
\forall \eta \in \Omega\left(\tau, h^{\prime}, \nu^{\prime}\right) \exists g \in \mathcal{S}\left(\tau, h^{\prime}, \eta\right):(\xi, g, \eta) \in \mathbf{A}^{\gamma}(\mathcal{N}) \forall \xi \in \mathbf{I}_{\tau} .
$$

By the definition of $\mathbf{A}$ (see (2.2)), relations (3.6), (3.8) mean that $\left(\tau, h^{\prime}, \nu^{\prime}\right) \in \mathbf{A}\left(\mathbf{A}^{\gamma}(\mathcal{N})\right)$. Hence, we have again $\left(\tau, h^{\prime}, \nu^{\prime}\right) \in \mathbf{A}^{\alpha}(\mathcal{N})$.

4. Thus, by virtue of the principle of transfinite induction, implication (3.5) holds for any ordinal $\eta$. Since the triples $(\tau, h, \nu)$ and $\left(\tau, h^{\prime}, \nu^{\prime}\right)$ are included in the conditions of Lemma 5 symmetrically, the state of the lemma follows from (3.5).

\subsection{Proof of Lemma 1}

1. Let $(t, x, \omega) \in \mathbf{A}^{\sigma}(\mathcal{N})$. Then, $(t, x, \omega) \in \mathbf{S P}_{\left(t_{0}, x_{0}\right)}$. Denote $\alpha \triangleq \Pi\left(\cdot \mid(t, x, \omega), \mathbf{A}^{\sigma}(\mathcal{N})\right)$. By the definition (see (2.1)), we have

$$
\begin{gathered}
\alpha \in \prod_{\nu \in \Omega(t, x, \omega)} \mathcal{P}(\mathcal{S}(t, x, \nu)), \\
(\tau, h, \eta) \in \mathbf{A}^{\sigma}(\mathcal{N}) \quad \forall \tau \in \mathbf{I} \forall h \in \alpha(\eta) \forall \eta \in \Omega(t, x, \omega) .
\end{gathered}
$$

In view of (3.2), we have $(t, x, \omega) \in \mathbf{A}\left(\mathbf{A}^{\sigma}(\mathcal{N})\right)$. Then, $\Pi\left(\nu \mid(t, x, \omega), \mathbf{A}^{\sigma}(\mathcal{N})\right) \neq \varnothing \forall \nu \in \Omega(t, x, \omega)$. So (see (3.9)), we get

$$
\alpha \in \prod_{\nu \in \Omega(t, x, \omega)} \mathcal{P}^{\prime}(\mathcal{S}(t, x, \nu))
$$

2. Suppouse $\nu, \nu^{\prime} \in \Omega(t, x, \omega)$ and $\theta \in \mathbf{I}_{t}$ satisfy the equality

$$
\left(\mathcal{S}\left(t_{0}, x_{0}, \nu\right) \mid \mathbf{I}^{\theta}\right)=\left(\mathcal{S}\left(t_{0}, x_{0}, \nu^{\prime}\right) \mid \mathbf{I}^{\theta}\right) .
$$

We show the embedding $\Gamma \subset \Gamma^{\prime}$ for the sets $\Gamma \triangleq\left(\alpha(\nu) \mid \mathbf{I}^{\theta}\right), \Gamma^{\prime} \triangleq\left(\alpha\left(\nu^{\prime}\right) \mid \mathbf{I}^{\theta}\right)$. 
Let $\gamma \in \Gamma$, then, the equality $\gamma=\left(h \mid \mathbf{I}^{\theta}\right)$ is true for some $h \in \alpha(\nu)$. Let us verify that

$$
(\theta, h, \nu) \in \mathbf{S P}_{\left(t_{0}, x_{0}\right)}, \quad \nu^{\prime} \in \Omega(\theta, h, \nu)
$$

By the choice of $h$, we have (see (3.11)) $h \in \mathcal{S}(t, x, \nu)$. From this inclusion, taking into account the relations $x \in \mathcal{S}\left(t_{0}, x_{0}, \omega\right), \nu \in \Omega(t, x, \omega)$ and property (1.4) of the system, we get $h \in \mathcal{S}\left(t_{0}, x_{0}, \nu\right)$. In particular (see (1.5)), we get the first inclusion in (3.13). Then, the second inclusion in (3.13) is well defined and is fulfilled in virtue of (1.6) and (3.12). According to inclusions (3.10), we have $(\theta, h, \nu) \in \mathbf{A}^{\sigma}(\mathcal{N})$. From the inclusion and the equality $\mathbf{A}^{\sigma}(\mathcal{N})=\mathbf{A}\left(\mathbf{A}^{\sigma}(\mathcal{N})\right)$ (Lemma 4), we obtain $(\theta, h, \nu) \in \mathbf{A}\left(\mathbf{A}^{\sigma}(\mathcal{N})\right)$. Hence (see $\left.(2.2)\right)$, the relation $\Pi\left(\eta \mid(\theta, h, \nu), \mathbf{A}^{\sigma}(\mathcal{N})\right) \neq \varnothing$ is true for all $\eta \in \Omega(\theta, h, \nu)$. In particular, taking into account the second inclusion in (3.13), we get the relation $\Pi\left(\nu^{\prime} \mid(\theta, h, \nu), \mathbf{A}^{\sigma}(\mathcal{N})\right) \neq \varnothing$. Let us choose some element $h^{\prime} \in \Pi\left(\nu^{\prime} \mid(\theta, h, \nu), \mathbf{A}^{\sigma}(\mathcal{N})\right)$. Then $($ see $(2.1))$,

$$
\left(\tau, h^{\prime}, \nu^{\prime}\right) \in \mathbf{A}^{\sigma}(\mathcal{N}) \quad \forall \tau \in \mathbf{I}_{\theta}
$$

and

$$
h^{\prime} \in \mathcal{S}\left(\theta, h, \nu^{\prime}\right)
$$

It follows from (1.1) and (3.15) that

$$
h^{\prime} \in \mathrm{Z}_{0}\left(h \mid \mathbf{I}^{\theta}\right) .
$$

Let us verify the relations

$$
\left(\tau, h^{\prime}, \nu^{\prime}\right) \in \mathbf{A}^{\sigma}(\mathcal{N}) \quad \forall \tau \in \mathbf{I}_{t}^{\theta} .
$$

Fix any $\tau \in \mathbf{I}_{t}^{\theta}$. Then, from (3.16), (3.12) and the choice of $h$, we get $(\tau, h, \nu) \in \mathbf{A}^{\sigma}(\mathcal{N})$, $\left(h \mid \mathbf{I}^{\tau}\right)=\left(h^{\prime} \mid \mathbf{I}^{\tau}\right),(\tau, h, \nu),\left(\tau, h^{\prime}, \nu^{\prime}\right) \in \mathbf{S P}_{\left(t_{0}, x_{0}\right)}, \Omega(\tau, h, \nu)=\Omega\left(\tau, h^{\prime}, \nu^{\prime}\right)$ by Lemma 3. From these relations, by Lemma 5, we get $\left(\tau, h^{\prime}, \nu^{\prime}\right) \in \mathbf{A}^{\sigma}(\mathcal{N})$. Since the choice of $\tau$ was arbitrary, (3.17) holds. Combining (3.14) and (3.17), we obtain

$$
\left(\tau, h^{\prime}, \nu^{\prime}\right) \in \mathbf{A}^{\sigma}(\mathcal{N}) \quad \forall \tau \in \mathbf{I}_{t}
$$

Using $(t, x, \omega) \in \mathbf{S P}_{\left(t_{0}, x_{0}\right)}$ and $\nu, \nu^{\prime} \in \Omega(t, x, \omega)$, by Lemma 3 , we get inclusions $(t, x, \nu),\left(t, x, \nu^{\prime}\right) \in$ $\mathbf{S P}_{\left(t_{0}, x_{0}\right)}$. Then, there exist $y, y^{\prime} \in \mathrm{Z}_{0}\left(x \mid \mathbf{I}^{t}\right)$ such that $y \in \mathcal{S}\left(t_{0}, x_{0}, \nu\right)$ and $y^{\prime} \in \mathcal{S}\left(t_{0}, x_{0}, \nu^{\prime}\right)$. From (3.12), using (1.2) and (3.1), we obtain

$$
\begin{gathered}
\left(\mathcal{S}(t, x, \nu) \mid \mathbf{I}^{\theta}\right)=\left(\mathcal{S}(t, y, \nu) \mid \mathbf{I}^{\theta}\right)=\left(\mathcal{S}\left(t_{0}, x_{0}, \nu\right) \cap \mathrm{Z}_{0}\left(y \mid \mathbf{I}^{t}\right) \mid \mathbf{I}^{\theta}\right) \\
=\left(\mathcal{S}\left(t_{0}, x_{0}, \nu^{\prime}\right) \cap \mathrm{Z}_{0}\left(y^{\prime} \mid \mathbf{I}^{t}\right) \mid \mathbf{I}^{\theta}\right)=\left(\mathcal{S}\left(t, y^{\prime}, \nu^{\prime}\right) \mid \mathbf{I}^{\theta}\right)=\left(\mathcal{S}\left(t, x, \nu^{\prime}\right) \mid \mathbf{I}^{\theta}\right) .
\end{gathered}
$$

From (3.15), (3.19), and $h \in \mathcal{S}(t, x, \nu)$, using (1.4), we get $h^{\prime} \in \mathcal{S}\left(t, x, \nu^{\prime}\right)$. The last inclusion and relations (2.1), (3.18) imply the inclusion $h^{\prime} \in \alpha\left(\nu^{\prime}\right)$. Combining it with (3.16), we get the relations $\gamma \triangleq\left(h \mid \mathbf{I}^{\theta}\right)=\left(h^{\prime} \mid \mathbf{I}^{\theta}\right) \in\left(\alpha\left(\nu^{\prime}\right) \mid \mathbf{I}^{\theta}\right)$. In other words, $\gamma \in \Gamma^{\prime}$. Because of arbitrary choice of $\gamma$, the embedding $\Gamma \subset \Gamma^{\prime}$ holds. Due to symmetry considerations, we have $\Gamma=\Gamma^{\prime}$. Since the choice of $\theta$, $\nu, \nu^{\prime}$ was arbitrary, we finally get that $\forall \nu, \nu^{\prime} \in \Omega(t, x, \omega) \forall \tau \in \mathbf{I}$

$$
\left(\left(\mathcal{S}\left(t_{0}, x_{0}, \nu\right) \mid \mathbf{I}^{\tau}\right)=\left(\mathcal{S}\left(t_{0}, x_{0}, \nu^{\prime}\right) \mid \mathbf{I}^{\tau}\right)\right) \Rightarrow\left(\left(\alpha(\nu) \mid \mathbf{I}^{\tau}\right)=\left(\alpha\left(\nu^{\prime}\right) \mid \mathbf{I}^{\tau}\right)\right)
$$

From (1.7), (3.11), and (3.20), we obtain $\alpha \in \mathbb{M}_{(t, x, \omega)}$ and, hence, (2.5). 


\subsection{Proof of Theorem 1}

From the relation $\mathbf{A}^{\sigma}(\mathcal{N}) \subset \mathcal{N}$ (see (2.4)), in view of Lemma 1, we find that, under the inequality $|\mathcal{N}| \prec \sigma$, the embedding below holds:

$$
\mathbf{A}^{\sigma}(\mathcal{N}) \subset\left\{(t, x, \omega) \in \mathcal{N} \mid \exists \alpha \in \mathbb{M}_{(t, x, \omega)}:(\tau, h, \nu) \in \mathcal{N} \forall \tau \in \mathbf{I}_{t} \forall h \in \alpha(\nu) \forall \nu \in \Omega(t, x, \omega)\right\} .
$$

1. Denote the set from the right-hand side of (2.6) by $\Lambda$. In view of (3.21), to prove the theorem, it is sufficient to establish the relation

$$
\Lambda \subset \mathbf{A}^{\sigma}(\mathcal{N})
$$

Since $\Lambda \subset \mathcal{N}$, we have

$$
\Lambda \subset \mathbf{A}^{0}(\mathcal{N})
$$

Let the ordinal $\zeta$ be such that, for all $\xi \prec \zeta$,

$$
\Lambda \subset \mathbf{A}^{\xi}(\mathcal{N})
$$

We show that $\Lambda \subset \mathbf{A}^{\zeta}(\mathcal{N})$. If $\zeta$ is a limit ordinal, then we obtain

$$
\Lambda \subset \bigcap_{\xi \prec \zeta} \mathbf{A}^{\xi}(\mathcal{N})=\mathbf{A}^{\zeta}(\mathcal{N})
$$

2. If $\zeta$ has a predecessor $\eta$, we shall verify that $\Lambda \subset \mathbf{A}\left(\mathbf{A}^{\eta}(\mathcal{N})\right)=\mathbf{A}^{\zeta}(\mathcal{N})$. Assume the contrary: there is a state $\left(t_{*}, x_{*}, \nu_{*}\right) \in \mathcal{N} \subset \mathbf{S P}_{\left(t_{0}, x_{0}\right)}$ such that

$$
\left(t_{*}, x_{*}, \nu_{*}\right) \in \Lambda \backslash \mathbf{A}^{\eta+1}(\mathcal{N}) .
$$

Then, it follows from (3.24) and (3.26) that $\left(t_{*}, x_{*}, \nu_{*}\right) \in \mathbf{A}^{\eta}(\mathcal{N}) \backslash \mathbf{A}\left(\mathbf{A}^{\eta}(\mathcal{N})\right)$. Hence, there exists $\omega^{*} \in \Omega\left(t_{*}, x_{*}, \nu_{*}\right)$ such that $\Pi\left(\omega^{*} \mid\left(t_{*}, x_{*}, \nu_{*}\right), \mathbf{A}^{\eta}(\mathcal{N})\right)=\varnothing$. From this equality, in view of the inclusion $\left(t_{*}, x_{*}, \nu_{*}\right) \in \mathbf{A}^{\eta}(\mathcal{N})$ and definition (2.1), we obtain

$$
\forall s \in \mathcal{S}\left(t_{*}, x_{*}, \omega^{*}\right) \quad \exists t \in \mathbf{I}_{t_{*}}: \quad\left(t, s, \omega^{*}\right) \notin \mathbf{A}^{\eta}(\mathcal{N}) .
$$

At the same time (see (3.26)), $\left(t_{*}, x_{*}, \nu_{*}\right) \in \Lambda$, and, hence, there exists a quasistrategy $\alpha_{*} \in$ $\mathbb{M}_{\left(t_{*}, x_{*}, \nu_{*}\right)}$ for which, by the definition of $\Lambda$,

$$
(t, s, \omega) \in \mathcal{N} \quad \forall t \in \mathbf{I}_{t_{*}} \quad \forall s \in \alpha_{*}(\omega) \quad \forall \omega \in \Omega\left(t_{*}, x_{*}, \nu_{*}\right) .
$$

In particular, as $\omega^{*} \in \Omega\left(t_{*}, x_{*}, \nu_{*}\right)$, we have

$$
\left(t, s, \omega^{*}\right) \in \mathcal{N} \quad \forall t \in \mathbf{I} \forall s \in \alpha_{*}\left(\omega^{*}\right) .
$$

Choose arbitrary $s^{*} \in \alpha_{*}\left(\omega^{*}\right)$. Then (see (1.7)), we have $s^{*} \in \mathcal{S}\left(t_{*}, x_{*}, \omega^{*}\right)$. According to (3.27), we have $\left(t^{*}, s^{*}, \omega^{*}\right) \notin \mathbf{A}^{\eta}(\mathcal{N})$ for some moment $t^{*} \in \mathbf{I}_{t_{*}}$. Hence, by $(3.24)$, we have $\left(t^{*}, s^{*}, \omega^{*}\right) \notin \Lambda$. In addition (see (3.29)), $\left(t^{*}, s^{*}, \omega^{*}\right) \in \mathcal{N}$ (and, therefore, $\left.\left(t^{*}, s^{*}, \omega^{*}\right) \in \mathbf{S P}_{\left(t_{0}, x_{0}\right)}\right)$. By the definition of $\Lambda$,

$$
\forall \alpha \in \mathbb{M}_{\left(t^{*}, s^{*}, \omega^{*}\right)} \quad \exists \omega \in \Omega\left(t^{*}, s^{*}, \omega^{*}\right) \quad \exists s \in \alpha(\omega) \quad \exists t \in \mathbf{I}_{t^{*}}: \quad(t, s, \omega) \notin \mathcal{N} .
$$

3. We define a multi-valued mapping $\beta \in \mathrm{Z}_{0}\left(s^{*} \mid \mathbf{I}^{t^{*}}\right)^{\Omega\left(t^{*}, s^{*}, \omega^{*}\right)}$ by the rule

$$
\beta(\omega) \triangleq \alpha_{*}(\omega) \cap \mathrm{Z}_{0}\left(s^{*} \mid \mathbf{I}^{t^{*}}\right) \quad \forall \omega \in \Omega\left(t^{*}, s^{*}, \omega^{*}\right) .
$$


We verify that $\beta$ is well defined: for any $\omega^{\prime} \in \Omega\left(t^{*}, s^{*}, \omega^{*}\right), \quad\left(\mathcal{S}\left(\left(t_{0}, x_{0}\right), \omega^{\prime}\right) \mid \mathbf{I}^{*}\right)=$ $\left(\mathcal{S}\left(\left(t_{0}, x_{0}\right), \omega^{*}\right) \mid \mathbf{I}^{t^{*}}\right)$ holds (see (1.6)). In view of $\omega^{*} \in \Omega\left(t_{*}, x_{*}, \nu_{*}\right)$ and (1.6), we have

$$
\left(\mathcal{S}\left(\left(t_{0}, x_{0}\right), \omega^{\prime}\right) \mid \mathbf{I}^{t_{*}}\right)=\left(\mathcal{S}\left(\left(t_{0}, x_{0}\right), \omega^{*}\right) \mid \mathbf{I}^{t_{*}}\right)=\left(\mathcal{S}\left(\left(t_{0}, x_{0}\right), \nu_{*}\right) \mid \mathbf{I}^{t_{*}}\right) .
$$

Wherefrom, we derive $\omega^{\prime} \in \Omega\left(t_{*}, x_{*}, \nu_{*}\right)$. As $\omega^{\prime}$ was chosen arbitrary, the embedding $\Omega\left(t^{*}, s^{*}, \omega^{*}\right) \subset$ $\Omega\left(t_{*}, x_{*}, \nu_{*}\right)$ holds. So, the mapping $\beta$ is well defined for all $\omega \in \Omega\left(t^{*}, s^{*}, \omega^{*}\right)$. Let us show the inclusion $\beta \in \mathbb{M}_{\left(t^{*}, s^{*}, \omega^{*}\right)}$.

For any $\nu \in \Omega\left(t^{*}, s^{*}, \omega^{*}\right)$ and $h \in \beta(\nu)$, by definition (3.31), we have $\left(h \mid \mathbf{I}^{t^{*}}\right)=\left(s^{*} \mid \mathbf{I}^{t^{*}}\right)$, $h \in \mathcal{S}\left(t_{*}, x_{*}, \nu\right)$. These relations by using of (1.3) and (1.2) imply $h \in \mathcal{S}\left(t^{*}, s^{*}, \nu\right)$. As $h$ and $\nu$ were chosen arbitrary, the inclusion $\beta \in \prod_{\chi \in \Omega\left(t^{*}, s^{*}, \omega^{*}\right)} \mathcal{P}\left(\mathcal{S}\left(t^{*}, s^{*}, \chi\right)\right)$ holds.

Let $\omega \in \Omega\left(t^{*}, s^{*}, \omega^{*}\right)$. We have (see (1.6)) $\left(\mathcal{S}\left(t_{0}, x_{0}, \omega\right) \mid \mathbf{I}^{t^{*}}\right)=\left(\mathcal{S}\left(t_{0}, x_{0}, \omega^{*}\right) \mid \mathbf{I}^{*}\right)$. Then, taking into account that $\alpha_{*} \in \mathbb{M}_{\left(t_{*}, x_{*}, \nu_{*}\right)}, \omega, \omega^{*} \in \Omega\left(t_{*}, x_{*}, \nu_{*}\right)$ and (1.7), we obtain $\left(\alpha_{*}(\omega) \mid \mathbf{I}^{t^{*}}\right)=$ $\left(\alpha_{*}\left(\omega^{*}\right) \mid \mathbf{I}^{\mathbf{t}^{*}}\right)$. By using the equality and the inclusion $s^{*} \in \alpha_{*}\left(\omega^{*}\right) \cap \mathrm{Z}_{0}\left(s^{*} \mid \mathbf{I}^{*}\right)$, we derive the relations

$$
\left(s^{*} \mid \mathbf{I}^{t^{*}}\right) \in\left(\alpha_{*}\left(\omega^{*}\right) \cap \mathrm{Z}_{0}\left(s^{*} \mid \mathbf{I}^{t^{*}}\right) \mid \mathbf{I}^{t^{*}}\right)=\left(\alpha_{*}(\omega) \cap \mathrm{Z}_{0}\left(s^{*} \mid \mathbf{I}^{t^{*}}\right) \mid \mathbf{I}^{t^{*}}\right)=\left(\beta(\omega) \mid \mathbf{I}^{t^{*}}\right) .
$$

So, the inequality $\left(\beta(\omega) \mid \mathbf{I}^{*}\right) \neq \varnothing$ holds. Hence, $\beta(\omega) \neq \varnothing$. In virtue of arbitrary choice of $\omega$, we have $\beta \in \prod_{\chi \in \Omega\left(t^{*}, s^{*}, \omega^{*}\right)} \mathcal{P}^{\prime}\left(\mathcal{S}\left(t^{*}, s^{*}, \chi\right)\right)$.

Concerning the second property of quasistrategies in (1.7) (non-anticipatory), the mapping $\beta$ inherits it from the quasistrategy $\alpha_{*}$. Indeed, by definition (see (3.31)), the mapping $\beta$ is an intersection of two non-anticipating mappings. So, $\beta \in \mathbb{M}_{\left(t^{*}, s^{*}, \omega^{*}\right)}$.

4. Then (see (3.30)),

$$
\exists \bar{\omega} \in \Omega\left(t^{*}, s^{*}, \omega^{*}\right), \exists \bar{s} \in \beta(\bar{\omega}), \exists \bar{t} \in \mathbf{I}_{t^{*}}: \quad(\bar{t}, \bar{s}, \bar{\omega}) \notin \mathcal{N} .
$$

But, by definition, $\bar{t} \in \mathbf{I}_{t_{*}}, \bar{\omega} \in \Omega\left(t_{*}, x_{*}, \nu_{*}\right)$ and $\bar{s} \in \alpha_{*}(\bar{\omega})$. In other words, (3.32) contradicts to (3.28). Hence, assumption (3.26) was wrong, and $\Lambda \subset \mathbf{A}\left(\mathbf{A}^{\eta}(\mathcal{N})\right)$. From the embedding, relations (3.23) and (3.25), on the basis of transfinite induction principle we get $\Lambda \subset \mathbf{A}^{\delta}(\mathcal{N})$ for any ordinal $\delta$. When $\delta=\sigma$, the embedding turns into desired relation (3.22).

\section{REFERENCES}

1. Chentsov A. G. On a game problem of converging at a given instant of time. Math. USSR-Sb., 1976. Vol. 28, No. 3. P. 353-376. DOI: 10.1070/sm1976v028n03abeh001657

2. Chentsov A. G. On a game problem of guidance with information memory. Dokl. Akad. Nauk SSSR, 1976. Vol. 227, No. 2. P. 306-309. (in Russian) http://mi.mathnet.ru/eng/dan40223

3. Engelking R. General Topology. Sigma Ser. Pure Math., vol. 6. Warszawa: Panstwowe Wydawnictwo Naukowe, 1985. 540 p.

4. Gomoyunov M.I., Serkov D. Control with a guide in the guarantee optimization problem under functional constraints on the disturbance. Proc. Steklov Inst. Math., 2017. Vol. 299, Suppl. 1. P. S49-S60. DOI: $10.1134 /$ S0081543817090073

5. Krasovskii N. N., Subbotin A. I. Game-Theoretical Control Problems. New York: Springer-Verlag, 1988. $517 \mathrm{p}$.

6. Ledyaev Y.S. Program-predictive feedback control for systems with evolving dynamics. IFACPapers OnLine, 2018. Vol. 51, No. 32. P. 723-726. DOI: 10.1016/j.ifacol.2018.11.461

7. Rockafellar R. T. Integrals which are convex functionals. Pacific J. Math., 1968. Vol. 24, No. 3. P. 525539. DOI: $10.2140 /$ pjm.1968.24.525

8. Serkov D. A. Transfinite sequences in the programmed iteration method. Proc. Steklov Inst. Math., 2018. Vol. 300, Suppl. 1. P. S153-S164. DOI: 10.1134/S0081543818020153

9. Serkov D. A., Chentsov A. G. The elements of the operator convexity in the construction of the programmed iteration method. Bull. South Ural State Univ. Ser. Math. Modell. Program. Comp. Software, 2016. Vol. 9, No. 3. P. 82-93. DOI: 10.14529/mmp160307 